\title{
Electrical Impedance Tomography: The Promise of Noninvasive Lung Images at the Bedside
}

Medical technology has been developing faster than many clinicians ever envisioned. One clear example is the revolution in the field of medical imaging. Early in development, it took time to get computed tomography (CT) images, and CT was less than ideal for the lungs because of respiratory movement. While lung images were taken, breath-holding was essential even in patients with respiratory failure. Now dynamic CT images are available.

Measurements of pressure and flow (volume) are the standard in the field of respiratory physiology. The major limitation of airway measurements are that each technique considers the lungs and airways as a single unit; local function cannot be evaluated. Volume delivered to the whole lung is evaluated, whereas local distribution is unavailable. Pressure to inflate the respiratory system is evaluated, but the local stress/strain of individual lung segments is not. When ARDS was reported in 1967 by Ashbaugh et al, ${ }^{1}$ chest radiographs were available; diffuse shadows on these images were characteristic of changes, and the lungs were considered to be damaged diffusely. The emergence of CT changed how ARDS was viewed. Now it is well recognized that inhomogeneous alveolar damage is a hallmark of ARDS. There have been many trials on interventions to improve ARDS outcomes, and lower tidal volume ventilation $^{2}$ and prone position ${ }^{3}$ have been shown to reduce mortality. Although several mechanisms of prone positioning were proposed, analysis of CT data revealed new edema development in the dependent lung and reabsorption of intra-alveolar edema in nondependent lung, free movement of interstitial fluid driven by gravity (sponge lung model), pulmonary blood redistribution resulting from gravity, and alveolar collapse in the dependent lung and alveolar reopening in nondependent lung. It appears that the shape-matching theory has the greatest chance to be a successful explanation for the mechanism of improved gas exchanged in the prone position. ${ }^{4}$ Thoracic CT has yielded tremendous information and has helped improve respiratory care. Without the knowledge achieved from CT images, the concept of

Correspondence: Masaji Nishimura MD PhD, Tokushima Prefectural Central Hospital, 1-10-3 Kuramoto, Tokushima, 770-8539 Japan. E-mail: nmasaji@tokushima-u.ac.jp.

DOI: $10.4187 /$ respcare. 07716 lung-protective strategies would have taken much longer to develop.

A weakness of CT is radiation exposure and the need to transport patients to radiology. Although dynamic images are available, it is generally limited to experi-

\section{See the Original Study on Page 281}

mental studies. Electrical impedance tomography (EIT) is a noninvasive, nonradiologic method of medical imaging. Conducting surface electrodes are attached to the skin above the tissue being examined, and small alternating currents are applied. The electrical conductivity, permittivity, and impedance of the tissue are inferred. It can theoretically be applied to any area, (eg, brain, cervix, breast, lungs, and muscle). The feasibility of EIT for lung imaging was first reported in $1990,{ }^{5}$ and the theory itself is not new. The resistivity of the lungs is much higher than that of other soft tissues in the thorax, which makes the lungs a good target for EIT monitoring.

X-rays travel linearly, while electric currents travel three-dimensionally along the path of lowest resistivity. Parts of electric currents are lost, and image construction in absolute EIT is complex. EIT was used experimentally for many years, and commercial versions are now becoming available. EIT does not require any kind of ionizing radiation, and currents are small enough not to stimulate peripheral nerves. EIT devices are smaller and less expensive than CT machines. Therefore, EIT has advantages compared to CT. Lung tissue conductance changes according to the content of air within alveoli, and EIT visualizes dynamic movement of the lungs. EIT also visualizes inhomogeneity of lung ventilation in mechanically ventilated patients at bedside. The images can be very impressive.

EIT also has some disadvantages. The Hounsfield number in CT images represents the linear relationship of the attenuation of water because the attenuation coefficient of air is nearly zero, and it reflects aeration of each part of the lungs. Time-difference EIT provides a relative difference of conductivity of each area. Color on screen does not necessarily reflect absolute aeration of each part. Another 


\section{EDITORIAL}

disadvantage of EIT is distinguished size. While small size tissues are distinguished with $\mathrm{CT}$ images, only tissues that are bigger than a given size can be detected with EIT, although this limitation depends on the number of electrodes used. Despite its disadvantages, the ability to monitor regional lung function at bedside with EIT renders these limitations acceptable. EIT is expected to give us incredible information on respiratory pathophysiology that was previously unavailable.

High-flow nasal cannula (HFNC) is simple and powerful device that has become very popular. ${ }^{6}$ While several physiological effects are presumed, the actual mechanism for each patient with a variety of pathophysiologic conditions remains to be clarified. In this issue of RESPIRATORY CARE, Yuan et $\mathrm{al}^{7}$ report on the effects of oxygen-delivery devices and body position on regional lung function with EIT in subjects extubated after abdominal surgery. HFNC improved end-expiratory lung impedance compared to conventional oxygen therapy both in ventral and dorsal lung regions. Head-up tilt improved end-expiratory lung impedance in the dorsal regions. Although the results are as expected, the effect of head-up tilt is much larger than HFNC. Though simple, this important information teaches us that patients should not be kept in a supine position.

Again, technology has been developing more quickly than many envisioned. Even with the current limitations of EIT, I anticipate that even more advanced EIT monitoring will be available in near future. EIT should give us an enormous amount of information and help improve patient care in the ICU.

Masaji Nishimura

Tokushima Prefectural Central Hospital

Tokushima, Japan

\section{REFERENCES}

1. Ashbaugh DG, Bigelow DB, Petty TL, Levine BE. Acute respiratory distress in adults. Lancet 1967;2(7511):319-323.

2. Brower RG, Matthay MA, Morris A, Schoenfeld D, Thompson BT, Wheeler A. Ventilation with lower tidal volumes as compared with traditional tidal volumes for acute lung injury and the acute respiratory distress syndrome. N Engl J Med 2000;342(18):1301-1308.

3. Guérin C, Reignier J, Richard JC, Beuret P, Gacouin A, Boulain T, et al. Prone positioning in severe acute respiratory distress syndrome. N Engl J Med 2013;368(23):2159-2168.

4. Gattinoni L, Pesenti A, Carlesso E. Body position changes redistribute lung computed-tomographic density in patients with acute respiratory failure: impact and clinical fallout through the following 20 years. Intensive Care Med 2013;39(11):1909-1915.

5. Cheney M, Isaacson D, Newell JC, Simske S, Goble J. NOSER: an algorithm for solving the inverse conductivity problem. Int J Imaging Syst Technol 1990;2(2):66-75.

6. Nishimura M. High-flow nasal cannula oxygen therapy in adults: physiological benefits, indication, clinical benefits, and adverse effects. Respir Care 2016;61(4):529-541.

7. Yuan Z, Han X, Wang L, et al. Oxygen therapy delivery and body position effects measured with electrical impedance tomography. Respir Care 2020;65(3):281-287. 